\title{
Structural Network Properties of Niche-Overlap Graphs
}

\author{
Nayla Sokhn* ${ }^{*}$, Richard Baltensperger ${ }^{\dagger}$, Louis-Felix Bersier ${ }^{\ddagger}$, Ulrich-Ultes Nitsche* and Jean Hennebert ${ }^{* \dagger}$ \\ $\dagger$ University of Applied Sciences of Western Switzerland, Switzerland \\ Email: nayla.sokhn@hefr.ch, richard.baltensperger@hefr.ch, jean.hennebert@hefr.ch \\ * Departement of Computer Science, University of Fribourg, Switzerland \\ Email: nayla.sokhn@unifr.ch, ulrich-ultes.nitsche@unifr.ch,jean.hennebert@unifr.ch \\ $\ddagger$ Departement of Ecology and Evolution, University of Fribourg, Switzerland \\ Email: louis-felix.bersier@unifr.ch
}

\begin{abstract}
The structure of networks has always been interesting for researchers. Investigating their unique architecture allows to capture insights and to understand the function and evolution of these complex systems. Ecological networks such as food-webs and niche-overlap graphs are considered as complex systems. The main purpose of this work is to compare the topology of 15 real niche-overlap graphs with random ones. Five measures are treated in this study: (1) the clustering coefficient, (2) the betweenness centrality, (3) the assortativity coefficient, (4) the modularity and (5) the number of chordless cycles. Significant differences between real and random networks are observed. Firstly, we show that niche-overlap graphs display a higher clustering and a higher modularity compared to random networks. Moreover we find that random networks have barely nodes that belong to a unique subgraph (i.e betweenness centrality equal to 0 ) and highlight the presence of a small number of chordless cycles compared to real networks. These analyses may provide new insights in the structure of these real niche-overlap graphs and may give important implications on the functional organization of species competing for some resources and on the dynamics of these systems.
\end{abstract}

Keywords: Food-webs, Niche-Overlap Graphs, Structure of Networks, Clustering Coefficient, Betweenness Centrality, Assortativity, Modularity, Chordless Cycles.

\section{INTRODUCTION}

The science of complex systems has accomplished a substantial improvement after the discovery of many topological properties that reveal the structure of these graphs. Understanding this structure helps the scientist explain the function and growth of these networks. The current field of studies includes social networks [1], world wide web networks [2], [3], scientific collaborators [4], mutualistic networks [5] and food-webs [6]. Researchers studied these graphs in order to try to reveal important patterns that are crucial for interpreting their behavior. For example, a better understanding of the structure of food-webs has potential implications in explaining the dynamics of ecological communities. Milo et al. compared the appearance of motifs in ecological networks with other networks [7]. Dunne et al. explored the relationship of species richness and other measures of complexity to ecosystem properties [8]. Moreover they found that food-webs display neither « small world » nor « scale-free » properties. A graph satisfies the small-world property if it exhibits a low diameter and a high clustering coefficient compared to random networks [9]. On the other hand, if a graph includes nodes with a very high number of links (called hubs), the graph then satisfies the scale-free property. Cattin et al. proposed a new model built on the hypothesis that any species' diet is the consequence of phylogenetic constraints and adaptation [10]. Banašek-Richter et al. found that larger webs are more richly endowed with the weak trophic interactions that recent theories show to be responsible for food-web stability [11]. Vermaat et al. showed that food webs are either clustered and highly interconnected or elongated with fewer links [12]. Williams et al. elaborated the probabilistic niche model that reveals the niche structure and the role of body size in foodwebs [13]. Rohr et al. developed a model to capture the architecture of food-webs to uncover the major factors underlying food-web organization [14]. Thierry et al. analysed the consequences of the body mass distribution for food web topology [15]. Naisbit et al. analysed a set of 13 foodwebs and proved the importance of phylogeny and body size on food web architecture [6]. Alcantara et al. created a framework based on the concept of strongly connected components, which are key structural components of networks necessary to explain stability properties such as persistence and robustness. They moreover suggest that ecological communities are not typically structured in multispecies compartments and that compartmentalization decreases robustness [16].

Although there have been many studies investigating the structure of food-webs and developing models to replicate their structure, to our knowledge no one yet explored the structure of niche-overlap graphs. These graphs are derived from foodwebs and describe the competition between predators. Two species are linked if they share at least one prey. In this paper, we extended the work introduced in [17] where it was shown that a collection of 15 niche-overlap graphs do not exhibit « small world » nor « scale-free » properties. We moreover compared their structure with random niche-overlap graphs to acquire a better comprehension of these patterns.

The rest of the paper is organized as follows. Section 2 introduces the ecological networks: food-webs and nicheoverlap graphs. Section 3 describes the network characteristics used to study the structure as well as the generated null models. Section 4 presents the results. Section 5 concludes and opens the discussion on potential future works. 


\section{FOOD-WEBS AND NICHE-OVERLAP GRAPHS}

\section{A. Graph representation}

Food-web shows who eat whom in natural communities. We represent it by drawing a directed graph where each node corresponds to a species and each directed link describes the flow of energy or biomass. From this graph, it is possible to create the niche-overlap graph which represents the competition graphs. Two predators are linked if they share at least one prey. Figure 1 illustrates a food-web graph and its associated niche-overlap graph.

A niche-overlap graph can be transformed to a weighted one by taking in consideration the number of common prey. The weight $\omega_{i, j}$ is defined by: $\omega_{i, j}=\frac{\mid \text { prey }_{i} \bigcap \text { prey }}{j} \mid$. In this paper, only binary niche-overlap graphs are treated. The analysis of weighted ones will be addressed in future work.
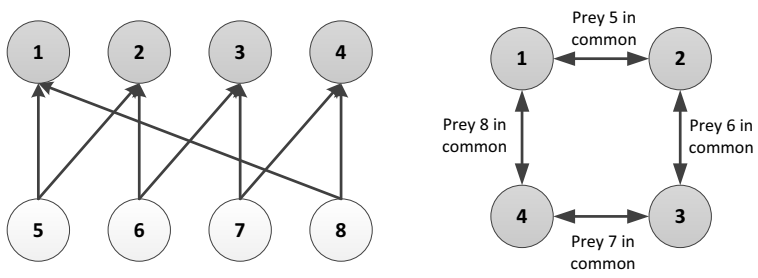

Fig. 1: Visualisation of a food-web graph of order 8 and its associated niche-overlap graph. The grey nodes are the predators and the white ones are the prey.

\section{B. Datasets}

We selected a collection of 15 real food-webs and generated their corresponding niche-overlap graphs (Table I) to conduct the analysis.

\begin{tabular}{ccccc}
\hline & \multicolumn{2}{c}{ Food-web } & \multicolumn{2}{c}{ Niche-overlap } \\
Graph & Order & Size & Order & Size \\
\hline Chesapeake & 33 & 71 & 27 & 95 \\
Cypdry & 68 & 468 & 53 & 855 \\
Cypress & 64 & 437 & 50 & 827 \\
Cypwet & 68 & 459 & 53 & 854 \\
Everglades & 63 & 617 & 58 & 1214 \\
Gramdry & 66 & 664 & 60 & 1267 \\
LRL North Spring 2 & 144 & 2095 & 111 & 2520 \\
LRL North Summer & 165 & 2706 & 121 & 3064 \\
LRL North Winter & 109 & 1257 & 86 & 1501 \\
LRL South Winter & 102 & 1328 & 83 & 1418 \\
LRL South Spring 1 & 151 & 2399 & 112 & 2965 \\
LRL South Summer & 173 & 2901 & 119 & 3652 \\
Saint Martin & 44 & 218 & 38 & 312 \\
Mangrovedry & 94 & 1210 & 86 & 2315 \\
Mangrove & 90 & 1151 & 84 & 2148 \\
\hline
\end{tabular}

TABLE I: Order (number of species) and size (number of links) for the selected food-webs and corresponding nicheoverlap graphs.

\section{NETWORK CHARACTERISTICS AND NUlL MODELS}

\section{A. Network characteristics}

In order to perform a network analyses on niche-overlap graphs, we selected four classical topological parameters to gain insights regarding the assemblage of species competing between others. We moreover enumerated the number of chordless cycles which give us an information on the intervality of these networks. The intervality shows the number of dimensions needed to define the niche of a species [18].

Clustering coefficient: the clustering coefficient is a ratio between the number of edges between the neighbors of $v$, and the maximum number of edges that could possibly exist between the neighbors of $v$. The clustering coefficient of the graph is the average of the clustering coefficient of each node. If the graph is fully connected, the clustering coefficient is then equal to 1 .

Betweenness centrality: the betweenness centrality of a node $v$ is the fraction of shortest paths in a graph that passes through $v$. A shortest path between two vertices is a path that minimises the number of edges that should be passed through in the graph to get from one node to the other.

By definition the betweenness centrality of a node $v$ is given by:

$$
B C(v)=\sum_{s, t \neq v} \frac{\sigma_{s t}(v)}{\sigma_{s t}},
$$

where $\sigma_{s t}$ is the total number of shortest paths from node $s$ to node $t$ and $\sigma_{s t}(v)$ is the number of shortest paths from $s$ to $t$ going through $v$ [19].

In [20] it is proved that the betweenness centrality of $v$ is equal to 0 if it belongs to only one complete subgraph of a graph $G$. By definition a complete subgraph is a part of a graph in which all vertices are connected to each other. The other trivial case is when $v$ has a degree of 1 .

Assortativity Coefficient: the assortativity coefficient $R$ of a graph is a correlation between the degree of two neighboring nodes [21]. It is defined as:

$$
R=\frac{\frac{1}{m} \sum_{i} j_{i} k_{i}-\left[\frac{1}{m} \sum_{i} \frac{1}{2}\left(j_{i}+k_{i}\right)\right]^{2}}{\frac{1}{m} \sum_{i} \frac{1}{2}\left(j_{i}^{2}+k_{i}^{2}\right)-\left[\frac{1}{m} \sum_{i} \frac{1}{2}\left(j_{i}+k_{i}\right)\right]^{2}},
$$

where $m$ is the size of the graph (number of edges) and $j_{i}, k_{i}$ are the degrees of the two endpoints of the ith edge, with $i=1, \ldots, m[21]$.

If $R$ is negative, the network is disassortative: high degree nodes are going to be connected to low degree nodes. Conversely, if $R$ is positive, The network is assortative: high degree nodes are going to be connected to high degree nodes. A value of $R$ equals to zero indicates that the connections between nodes are independent of node degree.

Modularity: the modularity $Q$ is a quality measure for graph clustering. It is used to reveal important network community structure. $Q$ ranging from 0 to 1 is defined by: 


$$
Q=\frac{1}{2 m} \sum_{i j}\left(A_{i j}-\frac{k_{i} k_{j}}{2 m}\right) \delta\left(c_{i}, c_{j}\right)
$$

where $m$ is the size of the graph (number of edges), $A$ is the adjacency matrix of the graph, $k_{i}, k_{j}$ is respectively the degree of node $i, j, c_{i}, c_{j}$ is respectively the community to which node $i, j$ is assigned and the function $\delta\left(c_{i}, c_{j}\right)$ is 1 if $c_{i}=c_{j}$ and 0 otherwise [22].

If $Q$ approaches 1, this indicates strong modular structure. If the number of intra-modular edges is no better than random, $Q=0$ [23].

Chordless cycle: A cycle $\left[v_{1}, v_{2}, \ldots, v_{k}\right](k>3)$ is a chordless one if it contains no chord. By definition a chord of a cycle is an edge between two vertices of the cycle that is not an edge of the cycle [24]. Figure 2 illustrates a chordal and chordless cycle respectively.

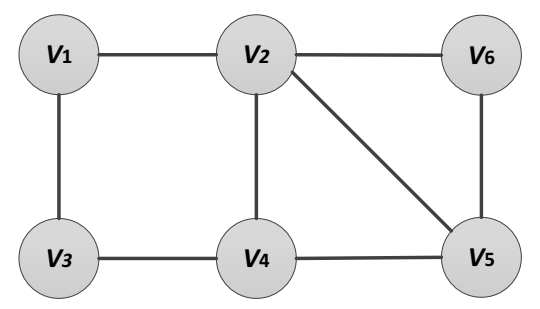

Fig. 2: In this network, $\left[v_{1}, v_{2}, v_{4}, v_{3}\right]$ is a chordless cycle and $\left[v_{2}, v_{6}, v_{5}, v_{4}\right]$ is a cycle with a chord.

\section{B. Null Models}

We generated two null models (100 simulations for each of the 15 graphs) to compare their structural properties described in Section III with the values obtained on the real graphs.

1) The first null model $\left(N_{1}\right)$ is the one introduced by Erdos and Renyi [25]. This model maintains the total number of links and shuffles them randomly.

2) The second one $\left(N_{2}\right)$ consists in swapping the links between species by keeping the sum of row and column of the adjacency matrix equals to the original one of the niche-overlap graph: the number of competitors for each species is conserved.

The first null model provides to what extent the observed network measures for real niche-overlap graphs depart from purely random networks. The second one shows if the identity of competitors for a given species has an influence on the network measures.

The null models were generated 100 times each. Therefore, we reported average values for the characteristics that were computed for these graphs.

\section{RESUlts AND Discussion}

Figure 3 illustrates the clustering coefficient for the real graphs and their null models.

The clustering coefficient of real graphs is higher compared to null models. A reasonable interpretation of this is that species tend to compete with species in clustered groups. Also notice that the clustering coefficient of the null model $N_{2}$ is higher than the $N_{1}$ model, this is explained by the fact that the initial number of competitors for each species was maintained for the model $N_{2}$.

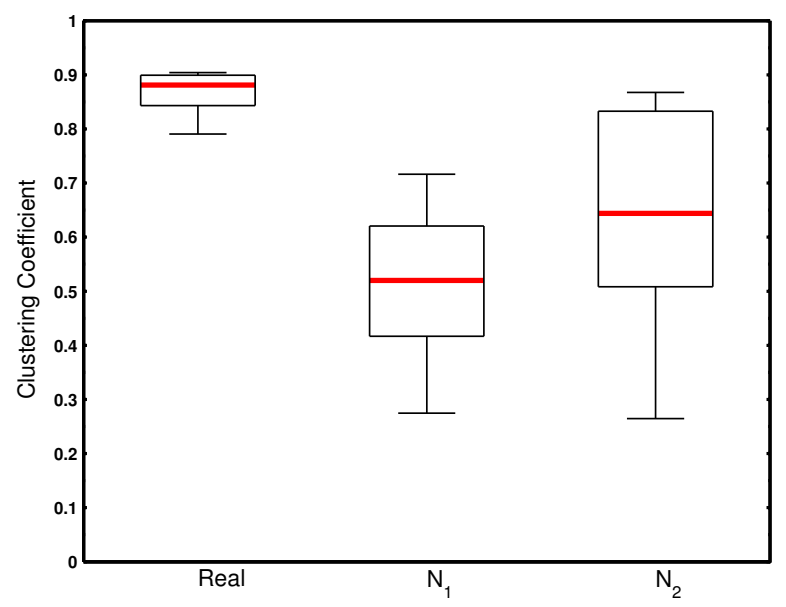

Fig. 3: The three box plots illustrate the distribution of the clustering coefficient for the real, $N_{1}$ and $N_{2}$ respectively.

Real graphs consist of a considerable percentage of species with a betweenness centrality equals to 0 . This reveals that some species tend to compete with only one particular group of species and hence belongs to a unique subgraph. Whereas for the null models, almost for all the graphs, species with a $B C$ equals to 0 is excluded or barely found (Table II).

Moreover the majority of these real graphs are neither assortative nor disassortative (neutral), almost all the values are very close to 0 (i.e non-assortative networks) (Table II). This indicates that species in these networks tend to be randomly linked, which goes in the same direction of a previous study where these networks were found to follow a single scale degree distribution [17].

The modularity of most of the real graphs is higher compared to the other two null models (Table III). This shows that species do not belong to one unique cluster. In fact, if $Q$ is equal to 0 all nodes belong to the same community, i.e. no community structure can be inferred from topological considerations. On the other hand, higher values of $Q$ indicate stronger community structure [26]. In this context, species within a cluster are most likely to be competing for the same resources and thus creating their own community.

Furthermore, real graphs include a small number of chordless cycles compared to both null models (Table III). This points out that real niche-overlap graphs have a bias toward intervality, i.e there is a way to order species so that almost all the resources of each consumers are adjacent in the or- 


\begin{tabular}{l|c|c|c||c|c|c}
\hline & \multicolumn{3}{|c|}{ \% of species with BC = 0 } & \multicolumn{3}{c}{ Assortativity } \\
\hline Graph & Real & $N_{1}$ & $N_{2}$ & Real & $N_{1}$ & $N_{2}$ \\
\hline Chesapeake & $70 \%$ & $1 \%$ & $0 \%$ & -0.12 & -0.08 & -0.09 \\
Cypdry & $19 \%$ & $0 \%$ & $2 \%$ & 0.19 & -0.04 & -0.16 \\
Cypress & $26 \%$ & $0 \%$ & $4 \%$ & 0.09 & -0.04 & -0.15 \\
Cypwet & $19 \%$ & $0 \%$ & $2 \%$ & 0.19 & -0.04 & -0.15 \\
Everglades & $14 \%$ & $0 \%$ & $1 \%$ & -0.04 & -0.04 & -0.17 \\
Gramdry & $12 \%$ & $0 \%$ & $1 \%$ & -0.04 & -0.03 & -0.16 \\
LRL North Spring 2 & $36 \%$ & $0 \%$ & $0 \%$ & 0.11 & -0.01 & -0.10 \\
LRL North Summer & $31 \%$ & $0 \%$ & $2 \%$ & 0.04 & -0.02 & -0.08 \\
LRL North Winter & $60 \%$ & $0 \%$ & $0 \%$ & 0.06 & -0.02 & -0.10 \\
LRL South Winter & $34 \%$ & $0 \%$ & $0 \%$ & 0.15 & -0.02 & -0.13 \\
LRL South Spring 1 & $38 \%$ & $0 \%$ & $0 \%$ & -0.07 & -0.02 & -0.13 \\
LRL South Summer & $16 \%$ & $0 \%$ & $0 \%$ & 0.09 & -0.02 & -0.14 \\
Saint Martin & $24 \%$ & $0 \%$ & $1 \%$ & -0.03 & -0.06 & -0.19 \\
Mangrovedry & $11 \%$ & $0 \%$ & $0 \%$ & -0.08 & -0.02 & -0.16 \\
Mangrove & $12 \%$ & $0 \%$ & $0 \%$ & -0.09 & -0.02 & -0.18 \\
\hline
\end{tabular}

TABLE II: Percentage of species belonging to one unique subgraph and assortativity's values of the niche-overlap graphs

\begin{tabular}{l|c|c|c||c|c|c}
\hline & \multicolumn{3}{|c||}{ Modularity } & \multicolumn{3}{c}{ Number of chordless cycles } \\
\hline Graph & Real & $N_{1}$ & $N_{2}$ & Real & $N_{1}$ & $N_{2}$ \\
\hline Chesapeake & 0.55 & 0.22 & 0.22 & 0 & 1778 & 1878 \\
Cypdry & 0.11 & 0.06 & 0.04 & 130 & 58996 & 2581 \\
Cypress & 0.08 & 0.05 & 0.04 & 0 & 32034 & 933 \\
Cypwet & 0.11 & 0.06 & 0.04 & 130 & 59258 & 2565 \\
Everglades & 0.06 & 0.04 & 0.03 & 710 & 44108 & 3760 \\
Gramdry & 0.07 & 0.04 & 0.03 & 1037 & 58735 & 5209 \\
LRL North Spring 2 & 0.32 & 0.08 & 0.07 & 25824 & 9549890 & 49486226 \\
LRL North Summer & 0.34 & 0.07 & 0.07 & 16904 & 6573835 & 88468714 \\
LRL North Winter & 0.33 & 0.09 & 0.08 & 360 & 78518333 & 7007851 \\
LRL South Winter & 0.36 & 0.09 & 0.08 & 224 & 27076680 & 2455460 \\
LRL South Spring 1 & 0.28 & 0.07 & 0.06 & 20789 & 137293917 & 32320747 \\
LRL South Summer & 0.23 & 0.06 & 0.05 & 36369 & 21030206 & 14669657 \\
Saint Martin & 0.21 & 0.12 & 0.10 & 356 & 20562 & 4584 \\
Mangrovedry & 0.15 & 0.05 & 0.04 & 29178 & 705268 & 228856 \\
Mangrove & 0.17 & 0.05 & 0.00 & 26179 & 729128 & 219971 \\
\hline
\end{tabular}

TABLE III: Modularity and number of chordless cycles of the niche-overlap graphs

dering and thus one dimension is sufficient to represent the community of predators [27].

\section{CONCLUSION}

In this paper, we investigated the structure of a collection of 15 niche-overlap graphs of highly resolved food-webs. We found that theses graphs are highly clustered and highly modular compared to random networks, moreover they include a considerable percentage of species with a betweenness centrality equals to 0 . Furthermore we found that real graphs are neither assortative nor disassortative and they include small number of chordless cycles. This work described here, represents a first analysis of the structure of niche-overlap graphs.

In future work, we will conduct an analysis on the structure of the weighted niche-overlap graphs. Additionally, we will run a Lotka-Volterra dynamical model on the 15 real niche-overlap graphs and on their null models $N_{1}$ and $N_{2}$ in order capture a better understanding on the persistence of species with regards to the architecture of these networks.

\section{REFERENCES}

[1] M. EJ. Newman and J. Park. Why social networks are different from other types of networks. Physical Review E, 68(3):036122, 2003.

[2] R. Albert, H. Jeong, and A. L. Barabási. Internet: Diameter of the world-wide web. Nature, 401(6749):130-131, 1999.

[3] A. L. Barabási, R. Albert, and H. Jeong. Scale-free characteristics of random networks: the topology of the world-wide web. Physica A: Statistical Mechanics and its Applications, 281(1):69-77, 2000.

[4] M. EJ. Newman. The structure of scientific collaboration networks. Proceedings of the National Academy of Sciences, 98(2):404-409, 2001.

[5] J. Bascompte. Structure and dynamics of ecological networks. Science, 329(5993):765-766, 2010.

[6] R. Naisbit, R. P. Rohr, A. G. Rossberg, P. Kehrli, and L.F. Bersier. Phylogeny versus body size as determinants of food web structure. Proceedings of the Royal Society B: Biological Sciences, 279(1741):32913297, 2012.

[7] R. Milo, S. Shen-Orr, S. Itzkovitz, N. Kashtan, D. Chklovskii, and U. Alon. Network motifs: simple building blocks of complex networks. Science Signalling, 298(5594):824, 2002.

[8] J. A. Dunne, R. J. Williams, and N. D. Martinez. Network structure and biodiversity loss in food webs: robustness increases with connectance. Ecology Letters, 5(4):558-567, 2002.

[9] D. J. Watts and S. H. Strogatz. Collective dynamics of small-world networks. nature, 393(6684):440-442, 1998. 
[10] M.F. Cattin, L.F. Bersier, C. Banašek-Richter, R. Baltensperger, and J.P. Gabriel. Phylogenetic constraints and adaptation explain food-web structure. Nature, 427(6977):835-839, 2004.

[11] C. Banašek-Richter, L.F. Bersier, M.F. Cattin, R. Baltensperger, J.P. Gabriel, Y. Merz, R. E. Ulanowicz, A. F. Tavares, D. D. Williams, P. C. Ruiter, et al. Complexity in quantitative food webs. Ecology, 90(6):1470-1477, 2009.

[12] J. E. Vermaat, J. A. Dunne, and A. J. Gilbert. Major dimensions in food-web structure properties. Ecology, 90(1):278-282, 2009.

[13] R. J. Williams, A. Anandanadesan, and D. Purves. The probabilistic niche model reveals the niche structure and role of body size in a complex food web. PloS one, 5(8):e12092, 2010.

[14] R. P. Rohr, H. Scherer, P. Kehrli, C. Mazza, and L.F. Bersier. Modeling food webs: exploring unexplained structure using latent traits. The American Naturalist, 176(2):170-177, 2010.

[15] A. Thierry, O. L. Petchey, A. P. Beckerman, P. H. Warren, and R. J. Williams. The consequences of size dependent foraging for food web topology. Oikos, 120(4):493-502, 2011.

[16] J. M. Alcántara and P. J. Rey. Linking topological structure and dynamics in ecological networks. The American Naturalist, 180(2):186199, 2012.

[17] Sokhn N., Baltensperger R., Hennebert J., Ultes-Nitsche U., and Bersier L.F. Structure analysis of niche-overlap graphs: Netsci2013. NetSci2013, 2013.

[18] José A Capitán, Alex Arenas, and Roger Guimerà. Degree of intervality of food webs: from body-size data to models. Journal of Theoretical Biology, 2013.

[19] L. C. Freeman. A set of measures of centrality based on betweenness. Sociometry, pages 35-41, 1977.

[20] R. Grassi, R. Scapellato, S. Stefani, and A. Torriero. Betweenness centrality: extremal values and structural properties. In Networks, Topology and Dynamics, pages 161-175. Springer, 2009.

[21] M. E.J. Newman. Assortative mixing in networks. Physical review letters, 89(20):208701, 2002.

[22] M. EJ. Newman. Analysis of weighted networks. Physical Review E, 70(5):056131, 2004.

[23] M. EJ. Newman and M. Girvan. Finding and evaluating community structure in networks. Physical review E, 69(2):026113, 2004.

[24] M. C. Golumbic. Algorithmic graph theory and perfect graphs, volume 57. North-Holland, 2004.

[25] P. Erdós and A. Rényi. On the evolution of random graphs. Publ. Math. Inst. Hungar. Acad. Sci, 5:17-61, 1960.

[26] V. Nicosia, G. Mangioni, V. Carchiolo, and M. Malgeri. Extending the definition of modularity to directed graphs with overlapping communities. Journal of Statistical Mechanics: Theory and Experiment, 2009(03):P03024, 2009.

[27] A. E. Zook, A. Eklof, U. Jacob, and S. Allesina. Food webs: Ordering species according to body size yields high degree of intervality. Journal of Theoretical Biology, 271(1):106-113, 2011. 